\title{
Quantum Gravity Microstates from Fredholm Determinants
}

\author{
Clifford V. Johnson $\odot^{*}$ \\ Department of Physics and Astronomy, University of Southern California, Los Angeles, California 90089-0484, USA
}

(Received 30 June 2021; revised 18 August 2021; accepted 8 October 2021; published 28 October 2021)

\begin{abstract}
Various two dimensional quantum gravity theories of Jackiw-Teitelboim (JT) form have descriptions as random matrix models. Such models, treated nonperturbatively, can give an explicit and tractable description of the underlying "microstate" degrees of freedom, which play a prominent role in regimes where the smooth geometrical picture of the physics is inadequate. This is shown using a natural tool, a Fredholm determinant $\operatorname{det}(\mathbf{1}-\mathbf{K})$, which can be defined explicitly for a wide variety of JT gravity theories. To illustrate the methods, the statistics of the first several energy levels of a nonperturbative definition of JT gravity are constructed explicitly using numerical methods, and the full quenched free energy $F_{Q}(T)$ of the system is computed for the first time. These results are also of relevance to quantum properties of black holes in higher dimensions.
\end{abstract}

DOI: 10.1103/PhysRevLett.127.181602

Introduction.-Jackiw-Teitelboim (JT) gravity [1] is a two dimensional model of gravity coupled to a scalar $\phi$, with Euclidean action on a spacetime manifold $\mathcal{M}$ :

$$
\begin{aligned}
I= & -\frac{1}{2} \int_{\mathcal{M}} \sqrt{g} \phi(R+2)-\int_{\partial \mathcal{M}} \sqrt{h} \phi_{b}(K-1) \\
& -\frac{S_{0}}{2 \pi}\left(\frac{1}{2} \int_{\mathcal{M}} \sqrt{g} R+\int_{\partial \mathcal{M}} \sqrt{h} K\right)
\end{aligned}
$$

where $g_{i j}$ is the metric with determinant $g, R$ is the Ricci scalar, $K$ is the trace of the extrinsic curvature for induced metric $h_{i j}$ on the boundary $\partial \mathcal{M}$, and $\phi_{b}$ is the boundary value of $\phi$. The constant $S_{0}$ multiplies the Einstein-Hilbert action, which yields the Euler characteristic $\chi(\mathcal{M})=$ $2-2 g-b, g$ counting handles and $b$ boundaries. The partition function of the full quantum gravity theory $Z(\beta)$ at some inverse temperature $\beta=1 / T$ is given by the path integral over $\mathcal{M}$ with a $\partial \mathcal{M}$ of length $\beta$. It has a topological expansion $Z(\beta)=\sum_{g=0}^{\infty} Z_{g}(\beta)$, where $Z_{g}(\beta)$ has a factor $e^{\chi(\mathcal{M}) S_{0}}$.

JT gravity is interesting not just as a solvable toy model of gravity, but also because it is a universal sector of the low $T$ near-horizon quantum dynamics of a wide class of higher dimensional black holes and branes [2], including ones in four dimensional asymptotically flat spacetimes. $S_{0}$ is the extremal $(T=0)$ Bekenstein-Hawking [3] entropy and $\phi$ parametrizes the leading finite $T$ deviation of the geometry

Published by the American Physical Society under the terms of the Creative Commons Attribution 4.0 International license. Further distribution of this work must maintain attribution to the author(s) and the published article's title, journal citation, and DOI. Funded by SCOAP ${ }^{3}$. from extremality. Insights about this model therefore directly pertain to important fundamental questions about quantum gravity and black holes in broader settings.

Solving the model (1) at leading (disc) order gives [4]

$Z_{0}(\beta)=\frac{e^{S_{0}} e^{\frac{\pi^{2}}{\beta}}}{4 \sqrt{\pi} \beta^{\frac{3}{2}}}, \quad \rho_{0}(E)=e^{S_{0}} \sinh (2 \pi \sqrt{E}) / 4 \pi^{2}$,

where the spectral density $\rho_{0}(E)$ comes from the Laplace transform: $Z_{0}(\beta)=\int_{0}^{\infty} \rho_{0}(E) e^{-\beta E} d E$. This model can be explicitly solved to any order to yield $Z_{g}(\beta)$ [also yielding corrections to $\left.\rho_{0}(E)\right]$. Correlations of multiple $Z(\beta)$ can also be obtained perturbatively. This was shown (for various types of JT gravity model) in Refs. [5,6], along with a striking equivalence (recalled below) to models of random $N \times N$ matrices at large $N$, where the topological expansion parameter $e^{-S_{0}} \sim 1 / N$.

This Letter's results go well beyond this topological expansion to uncover nonperturbative physics (building on a program of work begun in Ref. [7]) computing details of the individual underlying energy levels of the spectrum. This reveals the physics of the microscopic degrees of freedom of the quantum gravity theory: the underlying black hole microstates. The first six levels are shown in Fig. 1. For illustration, the plots are for $e^{-S_{0}}=1$. For a fixed reference value of $E$, smaller $e^{-S_{0}}$ (larger extremal entropy $S_{0} \sim \log N$ ) yields an increase in the number of levels found to the left, consistent with their microstate interpretation. A key observation is that the knowledge of the spectrum is fundamentally statistical, increasingly so at lower energies [8]. At higher $E$, the energy peaks become more sharply defined (their variance decreases), and also form a continuum. Here, the spacetime language (the perturbation theory described above) is a good 


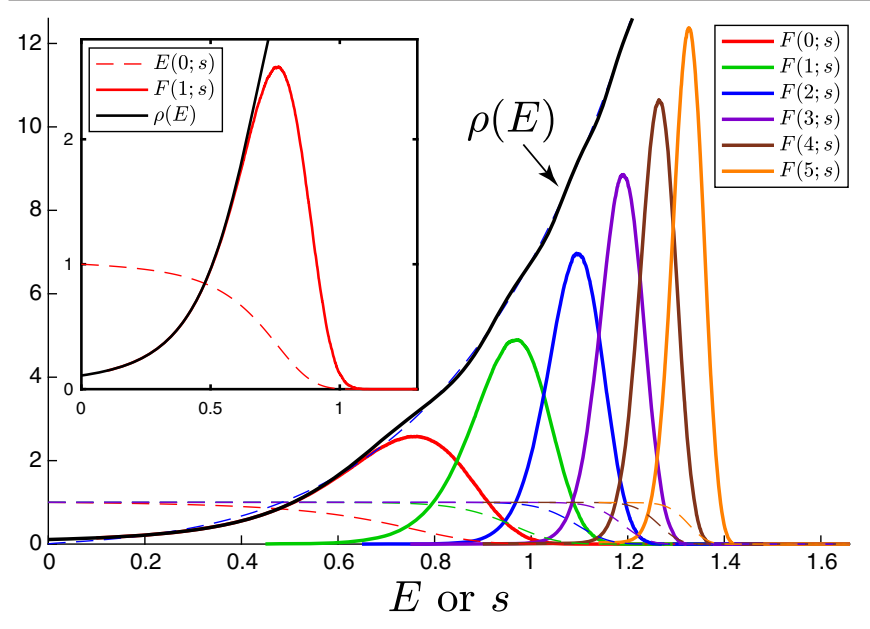

FIG. 1. Spectral density $\rho(E)$ (solid black), $\rho_{0}(E)$ (blue dashed), and probability densities (also cumulative probabilities, dashed) of the first six states of the JT gravity microstate spectrum. Inset: close-up of $\rho(E)$ and distributions for the ground state, with $\left\langle E_{0}\right\rangle \simeq 0.659$. Note that $\hbar=e^{-S_{0}}=1$ here.

approximation. Low $E$ is increasingly nonperturbative, a spacetime interpretation falls short, and the statistical description cannot be neglected: geometry and statistics are dual phenomena here.

Once the full spectrum and statistics are known, many key properties of the model can be computed. An example is the quenched free energy $F_{Q}(T)=-\beta^{-1}\langle\log Z(\beta)\rangle$, needed to compute thermodynamic quantities down to low $T$. Its computation has been discussed frequently [10-13], but so far not completed. Later in this Letter, $F_{Q}(T)$ will be computed for the first time.

Perturbative matrix models.-References [5,6] showed that the full topological expansion of a large class of models of JT gravity is captured by certain random matrix models in the "double-scaling limit" $[14,15]$. In the simplest matrix models, the probability distribution of the $N \times N$ matrix $M$ is $p(M)=e^{-\operatorname{Tr} V(M)}$ with Gaussian $V(M)=\frac{1}{2} M^{2}$ being the most famous prototype [17]. A more general polynomial $V(M)=\sum_{p} g_{p} M^{p}$ is of interest for studying gravity. (See e.g., Ref. [18] for a review.) Working at large $N$, the Feynman diagrammatic expansion of the matrix model partition function $\tilde{Z}=\int p(M) d M$ can be viewed [19] as tessellations of 2D Euclidean spacetimes, each order in the $1 / N$ expansion corresponding to the topology upon which the diagrams can be drawn. The double-scaling limit combines sending $N \rightarrow \infty$ with tuning the couplings $g_{p}$ to critical values such that surfaces large compared to the scale of the tessellation dominate, yielding universal continuum physics.

At large $N$ the matrix eigenvalues $\lambda_{i}$ can be described with a smooth coordinate $\lambda$. For an appropriate choice of $V(M)$, they occupy a finite segment of the real $\lambda$ line, with a spectral density $\rho(\lambda)$. The Laplace transform of $\rho(\lambda)$ is the expectation value of a "loop operator" $\left\langle\operatorname{Tr}\left[e^{-\ell M}\right]\right\rangle$, which makes a loop of length $\ell$ in the tessellations. The doublescaling limit focuses on the scaling region in the neighborhood of an end point [20] (say, $\lambda=\lambda_{0}$ ), magnifying it while sending the other end to infinity, i.e., $\lambda=\lambda_{0}+E \delta^{p}$, as parameter $\delta \rightarrow 0$, for some $p>0$, defining the scaled density $\rho(E)$.

In perturbation theory, everything at higher genus is determined by the leading (disc) order results by "topological recursion" properties [21] equivalent to certain "loop equations." Reference [5] showed that the JT gravity partition function $Z(\beta)$ is equivalent to a Hermitian matrix model loop expectation (of length $\beta$ ): at leading order, $\rho_{0}(E)$ was set as Eq. (2), with the parameter identification $e^{-S_{0}} \sim 1 / N$, and they proved that order by order the matrix model correlation functions that follow precisely match those obtained from the gravity path integral on manifolds $\mathcal{M}$, a highly nontrivial and remarkable result. However, nonperturbative contributions to $\rho(E)$ are beyond the reach of this recursive approach.

Nonperturbative matrix models. - The direct nonperturbative completion of Ref. [5]'s Hermitian matrix model definition of JT gravity has instabilities, which can be deduced [5,7] by noting that perturbatively it is a combination [22] (alternatively, a large $k$ limit [5]) of the family of $(2 k-1,2)$ "minimal string" models: the $k$ even sector was long known to be nonperturbatively unstable due to eigenvalue tunneling to infinite negative values. However Ref. [7] defined a nonperturbative completion of the JT gravity matrix model using double-scaled complex matrices $M$ for which the potential is built from $M M^{\dagger}$ [23]. It is a model of a Hermitian matrix with manifestly positive $\lambda$. Perturbatively identical to Ref. [5]'s model, it is nonperturbatively stable. [24].

The difference between the two types of models is visible in their orthogonal polynomial formulation. The standard Hermitian matrix model can be written entirely in terms of a system of $N$ polynomials $P_{i}(\lambda)$, orthogonal with respect to the measure $d \lambda e^{-V(\lambda)}$. They are related [e.g., for even $V(\lambda)$ ] according to $\lambda P_{i}(\lambda)=P_{i+1}(\lambda)+R_{i} P_{i-1}(\lambda)$. The $R_{i}$ satisfy a recursion relation determined by $V(\lambda)$. Computing the $R_{i}$ allows for the full solution of the matrix model [19]. As $N \rightarrow \infty, P_{i}(\lambda)$ and $R_{i}$ become functions of the coordinate $X=i / N \in[0,1]: P_{i}(\lambda) \rightarrow P(X, \lambda)$; $R_{i} \rightarrow R(X)$. After double scaling to an end point, scaled parts of all the key quantities survive to define the physics: $X, R(X)$, and $P(X, \lambda)$ have scaled pieces $x, u(x)$, and $\psi(x, E)$ (where $x \in \mathbb{R}$ ), and $R_{i}$ 's recursion relation becomes a differential equation for $u(x)$ that contains all the information about the model. The $\psi(x, E)$ are wave functions of an Hamiltonian that emerges as the scaled part of $\lambda$ (acting as an operator inside the matrix model integral):

$$
\mathcal{H}=-\hbar^{2} \frac{\partial^{2}}{\partial x^{2}}+u(x)
$$


Here, $\hbar$ is the scaling piece of the topological expansion parameter $1 / N$. Solving the double-scaled matrix model boils down to determining $u(x)$, defining a quantum mechanics problem from which everything can be computed:

$$
\mathcal{H} \psi(E, x)=E \psi(E, x) .
$$

In fact, the loop operator in this language is [26]

$$
Z(\beta)=\int_{-\infty}^{0} d x\left\langle x\left|e^{-\beta \mathcal{H}}\right| x\right\rangle .
$$

Putting $\int d \psi|\psi\rangle\langle\psi|=1$ into Eq. (5) and using Eq. (4) with $\psi \equiv\langle\psi \mid x\rangle$ gives $Z(\beta)=\int d E \rho(E) e^{-\beta E}$ with

$$
\rho(E)=\int_{-\infty}^{0}|\psi(E, x)|^{2} d x,
$$

i.e., knowing the complete $\psi(E, x)$ can be used to construct the full spectral density to all orders and beyond.

The Gaussian case $(k=1)$ illustrates the difference between the two types of model. In the Hermitian matrix model, the unscaled $P_{i}(\lambda)$ are Hermite functions. In the scaling limit they become [27] Airy functions $\psi(E, x)=\hbar^{-2 / 3} \mathrm{Ai}\left[-(E+x) \hbar^{-2 / 3}\right]$, which satisfy Eq. (4) for $u(x)=-x$ everywhere. Using them in Eq. (6) gives the full nonperturbative density: $\rho(E)=\hbar^{-2 / 3}\left[\mathrm{Ai}^{\prime}(\zeta)^{2}-\right.$ $\left.\zeta \operatorname{Ai}(\zeta)^{2}\right]$, with $\zeta \equiv-\hbar^{-2 / 3} E$, where $f^{\prime} \equiv \partial f / \partial \zeta$. Large $E$ gives the disc order result $\rho_{0}=E^{1 / 2} /(\pi \hbar)$, supported only on $E \geq 0$, while the full result is also nonzero on $E<0$. By contrast, for the complex matrix model, similar steps [23] give a nonlinear equation for $u(x)$ :

$$
u \mathcal{R}^{2}-\frac{\hbar^{2}}{2} \mathcal{R} \mathcal{R}^{\prime \prime}+\frac{\hbar^{2}}{4}\left(\mathcal{R}^{\prime}\right)^{2}=0,
$$

where $\mathcal{R}=u(x)+x$. The solution of interest has $u(x)=$ $-x$ for $x \rightarrow-\infty$ but $u(x)=0$ for $x \rightarrow+\infty$. So for large energies the behavior of the wave functions (in the $x<0$ regime, which corresponds to perturbation theory) is again like the "Airy model" form above. The physics is perturbatively identical. However the wave functions join on to a Bessel-like behavior for $x>0$, which becomes increasingly significant at low energies. The resulting fully nonperturbative $\rho(E)$ resembles that of the Airy model, but deviates at small $E$, truncating at $E=0$ [7].

There is an analogous model for any $k$ with this kind of behavior, and Refs. [7,28] showed how to build a nonperturbative completion of JT gravity using them. The equation for $u(x)$ is again of the form (7) but with a more complicated form for $\mathcal{R}$ (which is not needed here). Solving it to leading order gives a $u_{0}(x)$ that yields the JT spectral density (2), with $\hbar=e^{-S_{0}}$. Improving $u(x)$ order by order (expanding about large $x<0$ perturbation theory) yields the identical physics that Ref. [5] constructed. Reference [28] found (numerically, to good accuracy), the full solution for $u(x)$, determining $\mathcal{H}$, and then solved the spectral problem to yield wave functions $\psi(E, x)$. The integral (6) yielded the nonperturbative $\rho(E)$, plotted in Fig. 1 (solid black curve). At large $E$ it asymptotes to the classical result (2) (dashed line), but at small $E$ there are undulations that are invisible in perturbation theory, precursors of the underlying microphysics. [29].

Detailed microphysics.-It is possible to uncover much more, using new powerful tools. Fredholm determinants and their associated kernels have been widely used in the statistical physics literature to study random matrices [32]. In the scaling limit, the core object is the "kernel," built from the $\psi(x, E)$ as

$$
K\left(E, E^{\prime}\right)=\int_{-\infty}^{0} \psi(E, x) \psi\left(E^{\prime}, x\right) d x .
$$

In fact, its diagonal has already played a role, determining $\rho(E)$ in Eq. (6). Clearly there is a lot more information in its off-diagonal terms. It is the context of the Fredholm determinant construction that gives $K$ its kernel moniker. In problems of the form [33]: $f(E)-\int_{a}^{b} K\left(E, E^{\prime}\right) f\left(E^{\prime}\right) d E^{\prime}=$ $g(E)$, on some interval $(a, b)$ (or union of intervals) in the $E$ plane, the properties of $\operatorname{det}(\mathbf{I}-\mathbf{K})$ are important. $\mathbf{K}$ is the integral operator with kernel $K\left(E, E^{\prime}\right)$. [The random matrix and mathematics literature is filled with various kinds of kernel of interest (Airy, Bessel, sine, etc.). This Letter's studies of JT gravity now add some new kernels to the menagerie that also may be of interest.]

The Fredholm determinant can be used as follows: for the first energy level (labeled henceforth as the zeroth, for the ground state $E_{0}$ of the JT gravity system), the probability of finding no eigenvalues on the interval $(0, s)$ (chosen since the lowest possible energy is zero) is $E(0 ; s)=\operatorname{det}\left(\mathbf{I}-\left.\mathbf{K}\right|_{(0, s)}\right)$. This is a cumulative probability density function (CDF). The probability density function (PDF) for finding an energy is $F(0 ; s)=$ $-d E(0 ; s) / d s$. [For orientation, for the Airy model the interval would be $(-\infty, s)$, and using the Airy kernel yields the famous Tracy-Widom distribution [34] of the smallest eigenvalue. Here, the JT gravity system will reveal a new kind of smallest eigenvalue distribution.] For the $n$th energy level, the $\mathrm{CDF}$ on $(0, s)$ is (in a nonstandard notation)

$$
E(n ; s)=\sum_{j=0}^{n} \frac{(-1)^{j}}{j !} \frac{d^{j}}{d z^{j}} \operatorname{det}\left(\mathbf{I}-\left.z \mathbf{K}\right|_{(0, s)}\right) .\left.\right|_{z=1} .
$$

Correspondingly, the PDF is $F(n ; s)=-d E(n ; s) / d s$.

The major challenge now is to compute the determinant of this infinite dimensional operator. This requires much care, being prone to severe numerical difficulties even though the problem is effectively discrete ( $\sim 700$ energies 
were used). The impressive work of Ref. [35], showing how to use quadrature methods for such tasks, was extremely useful: using Clenshaw-Curtis quadrature to break up the interval into $m$ points $e_{i}$ and compute weights $w_{i}$ $(i=1 \cdots m)$, an integral $\int_{0}^{s} f(E) d E$ would be computed as $\sum_{i}^{m} w_{i} f\left(e_{i}\right)$. So similarly here

$\operatorname{det}\left(\mathbf{I}-\left.z \mathbf{K}\right|_{(0, s)}\right) \rightarrow \operatorname{det}\left[\delta_{i j}-z w_{i}^{1 / 2} K\left(e_{i}, e_{j}\right) w_{j}^{1 / 2}\right]$.

If the $\psi(E, x)$ are known very accurately (as for, e.g., Airy and Bessel kernels) the method gives impressive results with small values of $m$ such as 8 or 16 . Here, the $\psi(E, x)$ are numerical solutions to an eigenstate problem for which the potential $u(x)$ is itself a numerical solution to a (15th order [28]) nonlinear equation, so naturally some accuracy challenges were encountered. However, they can be surmounted well enough to get very good results for the JT gravity kernel, with $m=128$. [36].

The result for the zeroth level (the ground state $E_{0}$ ) is the focus of the inset of Fig. 1. A small amount of smoothing has been applied to remove numerical noise. To the left, at zero, the CDF $E(0 ; s)$ shows that there is a nonzero (but small) chance of finding $E_{0}$ there, falling steadily to zero to the far right with the increasing unlikelihood of finding $E_{0}$ at very high energies. The PDF $F(0 ; s)$ is also shown in the inset, peaking at $\sim 0.763$. This distribution's mean gives the average ground state of the ensemble as $\left\langle E_{0}\right\rangle \simeq 0.659$. Computing the results for higher levels is straightforward, although numerical inaccuracies in finding the first level get successively amplified with each level. The first six are shown in the main part of Fig. 1. What has been uncovered here with the Fredholm determinant technique are the explicit probability peaks for individual energy levels that, when added together, produce the previously found nonperturbative undulations in the spectral density (black line). Many key quantities can now be computed using this information, as will be demonstrated next [37].

Quenched free energy.- It is important to compute the quantity $F_{Q}(T)=-\beta^{-1}\langle\log Z(\beta)\rangle$ for JT gravity, but it is difficult, as discussed in Ref. [10], which also pointed out that connected diagrams with multiple boundaries ("replica wormholes") should play a crucial role. Reference [11] suggested that in addition a nonperturbative formulation was needed, e.g., a matrix model. There have been various useful partial results from toy models [12,13], at low $T$. The results for the full spectrum obtained here now makes computation of $F_{Q}(T)$ rather straightforward.

A numerical computation that simply directly samples the random matrix ensemble can be done in Gaussian cases, as done in Ref. [12]. However, the matrix model for JT gravity has exotic probability distributions, not readily simulated on a computer, so such an approach seems doomed here. Happily, the individual probability distributions for each level can now be generated by reverse engineering the new results for the $E(n ; s)$, which (recall)

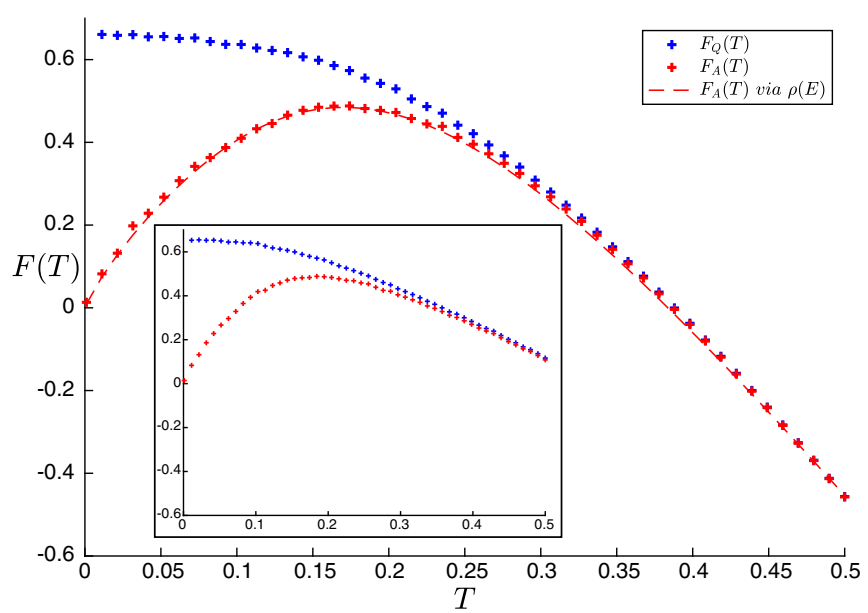

FIG. 2. The quenched and annealed free energies of JT gravity, computed using direct sampling (see text). As $T \rightarrow 0, F_{Q}(T)$ lands at $\left\langle E_{0}\right\rangle \simeq 0.659$. Here, $\hbar=e^{-S_{0}}=1$.

are CDFs. A key result from the theory of statistics is that samples from any PDF can be generated from uniform probability samples by mapping via the associated CDF. So, to build a representative sample partition function to use for ensemble averaging, generate the $n$th energy level $E_{n}$ with the appropriate probability [using uniform PDFs and mapping with the $\mathrm{CDF} E(n ; s)$ to sample the PDF $F(n ; s)]$. Then compute $\log [Z(\beta)]=\log \left(\sum_{n} e^{-\beta E_{n}}\right)$. This was done for an ensemble of just 5000 samples, and then averaged. For contrast, the annealed quantity $F_{A}(T)=$ $-\beta^{-1} \log \langle Z(\beta)\rangle$ can be computed too (averaging the partition function over the ensemble and taking the logarithm at the end).

Using just the first six levels yields the inset of Fig. 2. As higher levels are added, the details of the curves settle swiftly. Only successively higher $T$ details are affected by adding successively higher levels, the process asymptoting to the classical result at large $T$. In the main plot of Fig. 2 is a result with 150 levels. It is constructed by approximating the $E(n ; s)$ for levels above about $n=10$. In this regime the approximation is already very much under control. The point is that even by six levels (see Fig. 1), the peaks have narrowed and overlap significantly as the system returns to the continuum (classical) regime. The $n>10$ peaks are well approximated by narrow Gaussians, with their location, mean, and standard deviation determined by the classical curve (2). So their CDFs (error functions for Gaussians) were used for high levels. To test the computation, the red dashed line is the annealed $F_{A}(T)$ computed using the $Z(\beta)$ obtained by simply Laplace transforming $\rho(E)$ (integrated to the appropriate cutoff energy, the 150th level). The agreement with the result computed by direct ensemble averaging (red crosses) is remarkably good, confirming the accuracy of the result for $F_{Q}(T)$. Overall, rather nicely, $F_{Q}(T)$ is monotonically decreasing [i.e., the entropy $S(T)$ is manifestly positive], with zero slope at 
$T=0$, corresponding to entropy $S_{0}$ at extremality. This result also confirms Ref. [11]'s suggestion that the replica symmetry breaking transition conjectured in Ref. [10] does not occur.

Final remarks.-This Letter's results (with several JT supergravity examples to appear) demonstrate that random matrix models of JT gravity are completely tractable models of emergent geometry, a key quantum gravity phenomenon. There is a crossover from smooth spacetime geometry [large $E$ (or $T$ ) in Fig. 1 (or 2)] to a regime [small $E($ or $T)$ ] where spacetime is not enough, and a description in terms of individual discrete microstates (with wide variance) takes over. The key tool used here was the Fredholm determinant, built from the wave function $\psi(E, x)$. Interestingly, $\psi(E, x)$ has an interpretation [38] as a type of D-brane probe. So in a sense, the Fredholm tool is a D-brane probe that detects an increased spreading out or variance as it moves to lower energies. This is reminiscent of the D-brane probe involved in the "enhançon mechanism" [39] in higher dimensions, a connection worth exploring.

Finally, the microstates uncovered in detail here also model those of the higher dimensional black holes whose near-horizon low $T$ dynamics is controlled by a JT gravity. It will be interesting to see what other features of quantum gravity and black hole physics might be accessible using random matrix model technology.

*johnson1@usc.edu

[1] R. Jackiw, Nucl. Phys. B252, 343 (1985); C. Teitelboim, Phys. Lett. 126B, 41 (1983).

[2] A. Achucarro and M.E. Ortiz, Phys. Rev. D 48, 3600 (1993); P. Nayak, A. Shukla, R. M. Soni, S. P. Trivedi, and V. Vishal, J. High Energy Phys. 09 (2018) 048; K. S. Kolekar and K. Narayan, Phys. Rev. D 98, 046012 (2018); A. Ghosh, H. Maxfield, and G. J. Turiaci, J. High Energy Phys. 05 (2020) 104.

[3] J. D. Bekenstein, Phys. Rev. D 7, 2333 (1973); S. Hawking, Commun. Math. Phys. 43, 199 (1975).

[4] J. Maldacena and D. Stanford, Phys. Rev. D 94, 106002 (2016); J. Maldacena, D. Stanford, and Z. Yang, Prog. Theor. Exp. Phys. 2016, 12C104 (2016); K. Jensen, Phys. Rev. Lett. 117, 111601 (2016); J. Engelsöy, T. G. Mertens, and H. Verlinde, J. High Energy Phys. 07 (2016) 139.

[5] P. Saad, S. H. Shenker, and D. Stanford, arXiv:1903.11115.

[6] D. Stanford and E. Witten, Adv. Theor. Math. Phys. 24, 1475 (2020).

[7] C. V. Johnson, Phys. Rev. D 101, 106023 (2020).

[8] This Letter will not dwell on the interesting issue (discussed in Ref. [9], and now widely debated) of interpreting holographic correspondences when gravity seems to ensemble average over nongravitational duals.

[9] J. M. Maldacena and L. Maoz, J. High Energy Phys. 02 (2004) 053.

[10] N. Engelhardt, S. Fischetti, and A. Maloney, Phys. Rev. D 103, 046021 (2021).
[11] C. V. Johnson, arXiv:2008.13120.

[12] C. V. Johnson, arXiv:2104.02733.

[13] K. Okuyama, J. High Energy Phys. 12 (2020) 080; J. High Energy Phys. 03 (2021) 073; O. Janssen and M. Mirbabayi, J. High Energy Phys. 06 (2021) 074.

[14] E. Brezin and V. A. Kazakov, Phys. Lett. B 236, 144 (1990); M. R. Douglas and S. H. Shenker, Nucl. Phys. B335, 635 (1990); D. J. Gross and A. A. Migdal, Phys. Rev. Lett. 64, 127 (1990).

[15] This includes variants with nonorientable $\mathcal{M}$, and also supersymmetric extensions. Later work [16] extended the connection to models with more general potentials for $\phi$.

[16] H. Maxfield and G. J. Turiaci, J. High Energy Phys. 01 (2021) 118; E. Witten, Proc. R. Soc. A 476, 20200582 (2020).

[17] J. Wishart, Biometrika 20A, 32 (1928); E. P. Wigner, Ann. Math. 62, 548 (1955).

[18] P. H. Ginsparg and G. W. Moore, in Theoretical Advanced Study Institute (TASI 92): From Black Holes and Strings to Particles (World Scientific, Singapore, 1993), pp. 277469.

[19] G. 't Hooft, Nucl. Phys. B72, 461 (1974); E. Brezin, C. Itzykson, G. Parisi, and J. B. Zuber, Commun. Math. Phys. 59, 35 (1978); D. Bessis, C. Itzykson, and J. B. Zuber, Adv. Appl. Math. 1, 109 (1980).

[20] S. Dalley, C. V. Johnson, and T. Morris, Mod. Phys. Lett. A 06, 439 (1991); M. J. Bowick and E. Brezin, Phys. Lett. B 268, 21 (1991).

[21] M. Mirzakhani, Inventiones Mathematicae 167, 179 (2006); B. Eynard and N. Orantin, arXiv:0705.3600; E. Witten, Quart. J. Math. Oxford Ser. 72, 701 (2021).

[22] K. Okuyama and K. Sakai, J. High Energy Phys. 01 (2020) 156.

[23] T. R. Morris, Nucl. Phys. B356, 703 (1991); S. Dalley, C. V. Johnson, and T. Morris, Nucl. Phys. B368, 625 (1992); T. R. Morris, Classical Quant. Grav. 9, 1873 (1992).

[24] Other nonperturbative completions are proposed in, e.g., Refs. [5,25]. It would be interesting to investigate what features they may have in common with the completion presented in this Letter.

[25] P. Gao, D. L. Jafferis, and D. K. Kolchmeyer, arXiv:2104 .01184 .

[26] T. Banks, M. R. Douglas, N. Seiberg, and S. H. Shenker, Phys. Lett. B 238, 279 (1990).

[27] G. W. Moore, Prog. Theor. Phys. Suppl. 102, 255 (1990); P. Forrester, Nucl. Phys. B402, 709 (1993).

[28] C. V. Johnson, Phys. Rev. D 103, 046013 (2021).

[29] This method yielded explicit nonperturbative $\rho(E)$ for a large class of supersymmetric models in Refs. [11,28,30], as well as deformed JT gravity models and an additional supersymmetric model in Refs. [31].

[30] C. V. Johnson, Phys. Rev. D 103, 046012 (2021).

[31] C. V. Johnson and F. Rosso, J. High Energy Phys. 04 (2021) 030; C. V. Johnson, F. Rosso, and A. Svesko, Phys. Rev. D 104, 086019 (2021).

[32] M. L. Mehta, Random Matrices, 3rd ed. (Academic Press, Cambridge, 2004); P. Forrester, Log-Gases and Random Matrices (Princeton University Press, Princeton, 2010). 
[33] I. Fredholm, Acta Math. 27, 365 (1903).

[34] C. A. Tracy and H. Widom, Commun. Math. Phys. 159, 151 (1994).

[35] F. Bornemann, Math. Comput. 79, 871 (2010).

[36] The CHEBFUN package in MATLAB helped produce more refined $\psi(E, x)$ than were previously used in Refs. [7,11,28], giving a numerically cleaner $K\left(E, E^{\prime}\right)$.
[37] There are quantities (e.g., correlation functions) that require additional statistical information such as joint probabilities of levels. They are not considered here.

[38] J. M. Maldacena, G. W. Moore, N. Seiberg, and D. Shih, J. High Energy Phys. 10 (2004) 020.

[39] C. V. Johnson, A. W. Peet, and J. Polchinski, Phys. Rev. D 61, 086001 (2000). 